\title{
Cómo gestionar programas de digitalización en museos
}

\section{PREMIO CIMED}

\section{How to manage digitization programs in museums}

Full English versión at Anexo I

\section{Pere Viladot $^{\mathrm{a}}$, Marta Soler ${ }^{\mathrm{b}}$, Javier Hidalgo ${ }^{\mathrm{c}}$, Erik Stengler ${ }^{\mathrm{d}}$ y Guillermo Fernández}

${ }^{a}$ Ex-jefe del Departamento de Educación y Actividades del Museu de Ciències Naturals de Barcelona., pere@viladot.cat, ${ }^{\mathrm{b}}$ Museu Agbar de les Aigües, msolerar@laescueladelagua.com, ${ }^{\mathrm{C} F u n d a c i o ́ n ~ B a n c a r i a ~}$ La Caixa, cosmojavi@gmail.com, ${ }^{\mathrm{d}}$ Universidad del Estado de Nueva York en Oneonta (SUNY Oneonta), Erik.Stengler@oneonta.edu, y ${ }^{\mathrm{e}}$ consultor independiente para museos y exposiciones, info@lenguajemuseografico.com ${ }^{\text {abcde }}$ Colectivo el Museo Transformador

\begin{abstract}
Resumen
A lo largo de la pandemia de la COVID-19, hemos asistido a múltiples webinarios y actividades online, donde se ha puesto de manifiesto la necesidad de profundizar en la digitalización de los museos. Estos han suplido el cierre obligado por variadas propuestas en este sentido, en su mayor parte ya existentes o realizadas con urgencia y frecuentemente careciendo del contexto de una gestión estratégica adecuada.

Casi todos los debates han puesto de manifiesto que se acumula un retraso evidente en estos procesos y que, además, se debería aprovechar esta oportunidad para darle un nuevo impulso. El peligro es que se opte por una política ejecutiva y no estratégica, como suele pasar demasiado a menudo en los museos. Es decir, que se acabe dando prioridad a evidenciar alguna acción por parte del museo, antes que a diseñar un proyecto vinculado con la planificación estratégica a largo plazo. Por otra parte, no siempre se establece una separación conceptual entre la idea de ofrecer la versión digital de un museo o, sencillamente, perfeccionar su web.
\end{abstract}

Ante ello, como parte de la misión de El Museo Transformador, hemos desarrollado una herramienta de evaluación que ayude a tomar las decisiones de forma reflexiva y no sólo en contextos de emergencia. Estas decisiones no sólo deben tener en cuenta los aspectos tecnológicos, que a menudo deslumbran por cautivadores, sino sobre todo los aspectos de sostenibilidad en el tiempo.

La herramienta es una guía con criterios de evaluación pautados y definidos. Esta guía se compone de una introducción para evaluar la gestión estratégica y una rúbrica para evaluar el valor museístico y la gestión ejecutiva. La 
introducción sirve para conocer si una propuesta es adecuada: si impulsa nuestra misión, apoya los objetivos estratégicos y refuerza las áreas clave de nuestra organización. Para evaluar el valor museístico, la propuesta deberá ofrecer un vínculo claro con la experiencia museística, en sus valores educativos y en su relación con los contenidos propios del museo y el lenguaje museográfico. Finalmente, para la gestión ejecutiva, la propuesta debe tener en cuenta los recursos financieros, humanos, materiales y tecnológicos necesarios y si formula una propuesta de evaluación completa. Asimismo, debe definir el tipo de público al que se dirige y cómo se plantea su participación.

Palabras clave: Evaluación previa, programas digitales, museos, gestión estratégica, rúbrica

\footnotetext{
Abstract

Throughout the COVID-19 pandemic, we have attended multiple webinars and online activities that have highlighted the need to further develop museum digitization. Museum closings prompted various proposals to be offered, most of which were rehashed or put together in haste, often without proper strategic management.
}

Almost all the discussions have demonstrated that there is a delay in these processes and that this opportunity should be used to give new impetus to digitalization. The danger is that an executive policy could be chosen instead of a strategic one, as is often the case in museums. In other words, priority is given to demonstrating action rather than designing a project linked to longterm strategic planning. In addition, a conceptual separation is not always established between offering a digital version of a museum or simply improving its website.

As part of the mission of El Museo Transformador (The Transformative Museum), we have developed an evaluation tool that reflectively aids organizational decision-making, not only in emergency contexts. These decisions need to include technological aspects, which are often dazzlingly captivating, but especially that of sustainability over time.

The tool is a guide with established and defined evaluation criteria. It consists of an introduction for the assessment of strategic management and a rubric to assess museum value and executive management. The introduction serves to evaluate whether a proposal is appropriate in terms of promoting an organization's mission, supporting its strategic goals, and reinforcing key areas of the organization. To evaluate museum value, the proposal must offer a clear link with the museum experience in its educational values and its relationship with the museum's own contents and museographic language. 
Finally, in terms of executive management, the proposal must take into account the necessary financial, human, material, and technological resources and whether it formulates a complete evaluation strategy. The proposal must also define the target audience and participation mechanisms.

Keywords: Prior evaluation, digital programs, museums, strategic management, rubric. 


\section{Introducción}

La pandemia de la COVID-19 generó múltiples seminarios y jornadas web para debatir el futuro de los museos post-COVID, especialmente de la necesidad de avanzar hacia un museo más social, más transversal, más digital. Muchos museos, una vez cerrados, potenciaron su oferta a través de Internet para continuar conectados con su público. De ahí que hubiera un consenso unánime en que una vez pasada la pandemia no habría vuelta atrás.

Pero en la mayor parte de los casos, esta oferta no suponía más que una traslación del mundo analógico de lo presencial al mundo digital de lo online aportando poco más que un trabajo de optimización del sitio web del museo. Es obvio que el museo debe avanzar hacia la incorporación de la tecnología digital en sus múltiples facetas - redes sociales, 3D, videoblogs, etc.- - Pero ¿cómo? ¿Con qué criterios decidimos si una iniciativa es pertinente o no?

Demasiado a menudo, los museos actúan de forma compulsiva para demostrar que no se están quietos, que hacen algo. Y ante demandas más mediáticas que sociales, llenan su programación de actividades que poco tienen que ver con su core bussiness: el patrimonio y las exposiciones. ¿Para qué? ¿Con qué objetivos? ¿Con qué resultados? Un reciente artículo en Rolling Stone lo expresaba de forma diáfana (Brockër, 2021): «But how much of this innovation has been strategic? How much thinking is behind what is being built? Are these digital initiatives backed by the whole organization or were they the spontaneous brilliance of a specific team member or rogue technologist?». Poco se habla de ello.

Vista la situación, en el colectivo El museo Transformador (www.elmuseotransformador.org) nos propusimos aportar una herramienta de evaluación previa para ayudar a los museos en la toma de decisiones para desarrollar iniciativas digitales. Apostamos por un museo contemporáneo dedicado a producir transformaciones constatables en la cultura y la educación de la sociedad. Nos dimos a conocer en abril de 2020 en pleno confinamiento con base en un manifiesto que escribimos en enero de aquel año. En el momento de redactar esta comunicación, 55 personas ya se han adherido a nuestro manifiesto y tenemos casi 900 seguidores en Twitter.

\section{Objetivos}

Se estima que, a medio o largo plazo, la demanda de la cultura volverá a ser presencial, tanto en los escenarios más optimistas como en los más pesimistas como se puede ver en la figura 1 (López, 2021). Así lo vaticinábamos en un artículo que escribimos al principio de la pandemia (Stengler et. al., 2020). En él, planteábamos que, como afirmaba Nina Simon (2020), la producción acelerada de servicios digitales no era un síntoma de lo que había que hacer en el futuro. En palabras de Simon, «no dejemos que la producción sea enemiga de lo bueno». 


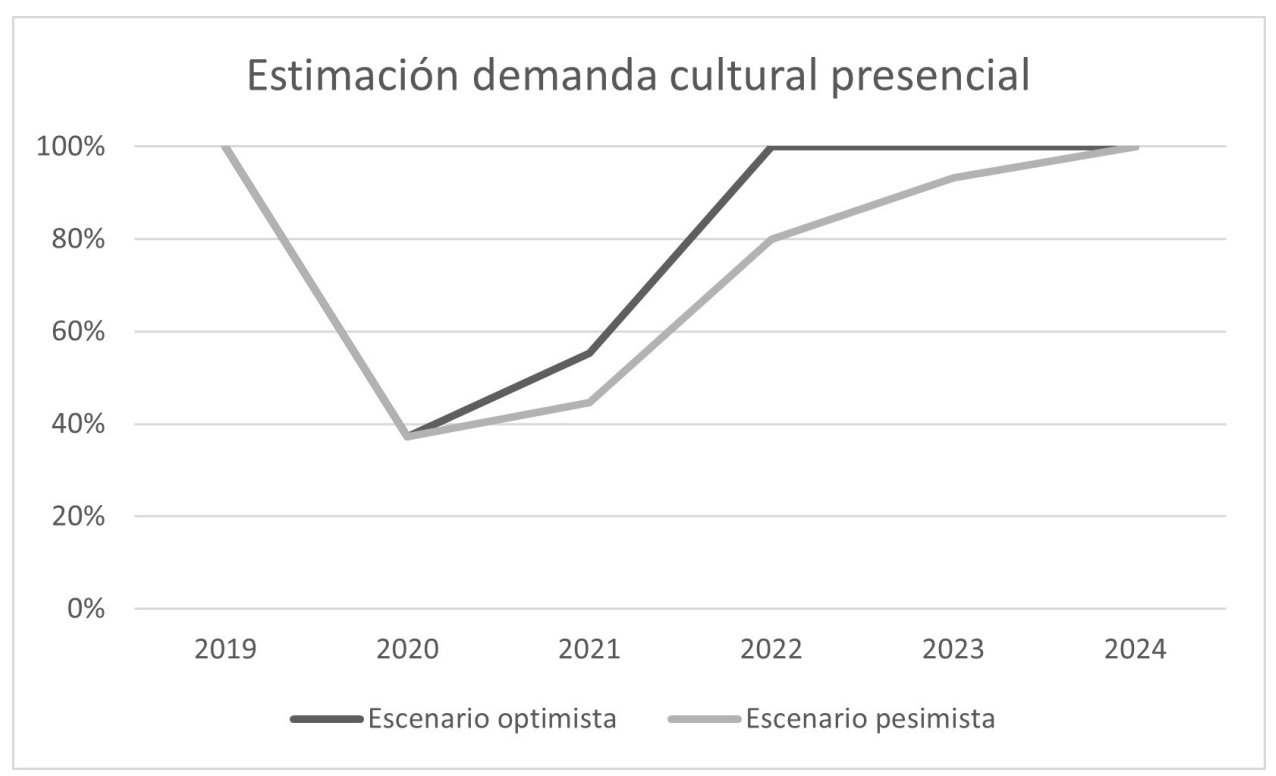

Fig.1 Estimación de la demanda cultural presencial en un escenario optimista y en uno pesimista.

Ante la falta de una reflexión a fondo de la estrategia digital a largo plazo, nuestro objetivo principal es el de ofrecer una herramienta evaluativa de reflexión, que ayude en la toma de decisiones estratégicas para el desarrollo de iniciativas de digitalización en los museos. Partimos de tres preguntas que cualquier propuesta debe responder antes de tomar cualquier decisión:

- ¿Impulsa nuestra misión?

- ¿Apoya los objetivos estratégicos?

- ¿Apuntala las áreas clave de nuestra organización?

La respuesta afirmativa sincera a estas preguntas debería hacer emerger otras dos que serán las que nos darán la medida de la pertinencia de la iniciativa:

- ¿Qué relación tiene con nuestros valores, contenidos y lenguaje museísticos?

- ¿Disponemos de recursos para abordarlo o hay que desvestir a un santo para vestir a otro?

Estas dos últimas preguntas, son las que desarrollamos en la rúbrica que presentamos. Así pues, los objetivos concretos de nuestra propuesta de herramienta evaluativa son:

1. Ayudar en la toma estratégica de decisiones con un instrumento de fácil aplicación.

2. Garantizar un proceso de reflexión transversal que implique a todos los departamentos. 
3. Potenciar la visión institucional de las propuestas.

\section{Desarrollo de la innovación}

\subsection{Qué es un museo}

Para poder dar respuesta a las preguntas que formulábamos en el apartado anterior, debemos plantearnos lo que es un museo. El museo contemporáneo ha pasado de finalidad a medio. Un medio que utiliza el lenguaje museográfico para comunicar sus relatos basados en el patrimonio que conserva y protege, con una evidente finalidad educativa, para promover la transformación social. El museo no expone objetos, sino que comunica hechos, sucesos, evidencias, expresiones naturales o humanas, es decir, manifestaciones perceptibles. En este sentido, clasificamos estas manifestaciones en tres categorías como puede verse en la figura 2 .

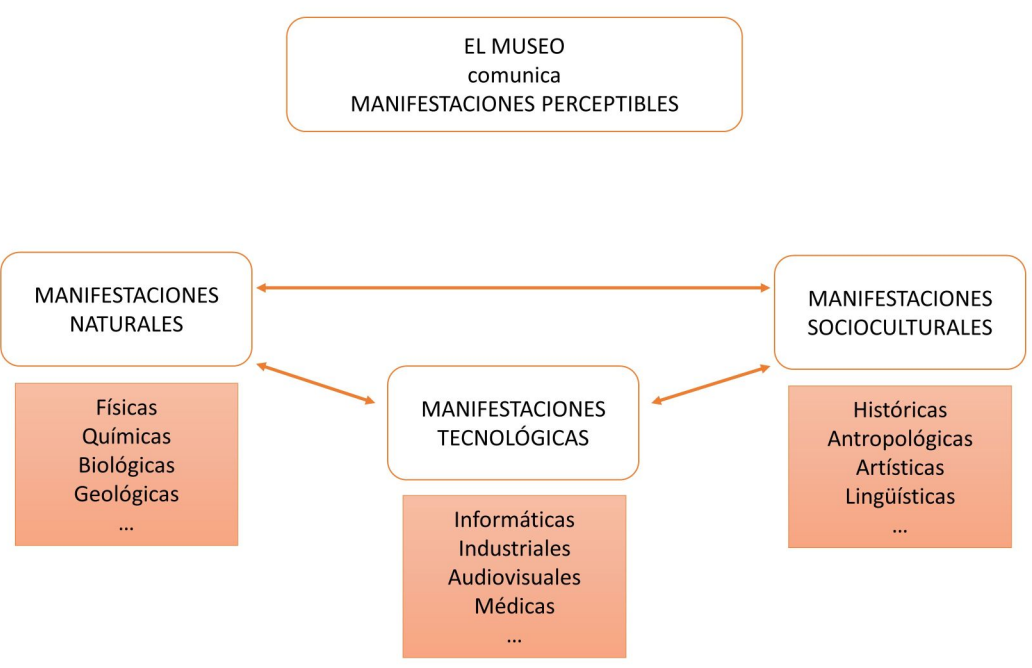

Fig. 2. EI museo comunica manifestaciones perceptibles que podemos clasificar en tres categorías: naturales, socioculturales y tecnológicas

Los museos comunican estas manifestaciones a través de narraciones de diversa índole que deberían ser el leitmotiv de cualquier museo. Si no hay relato, no hay comunicación posible. De manera explícita o implícita, los museos siempre han comunicado relatos a la sociedad. El poder de ciertas élites con base en la acumulación de objetos de arte, el espíritu expoliador colonialista de una raza superior, la acumulación de conocimiento por élites culturales, etc. En pleno siglo XXI cuando el museo debe tomar consciencia de su poder transformador crítico, el relato, las historias que conllevan estas manifestaciones, deben ser el núcleo central de los museos como medio de comunicación (véase la figura 3). 


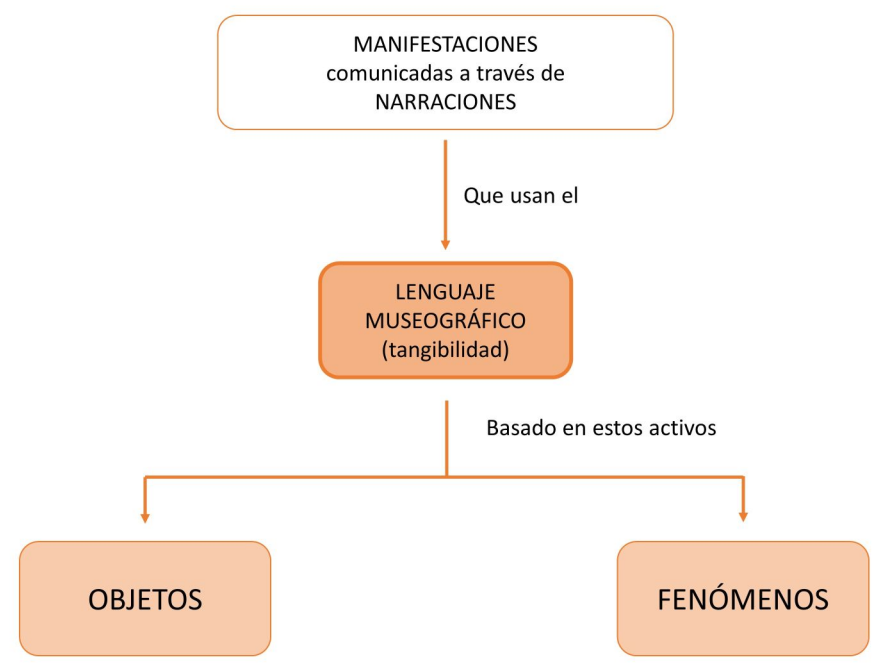

Fig. 3 Las manifestaciones perceptibles son comunicadas en el museo a través de narraciones que utilizan el lenguaje museográfico basado en objetos (auténticos o representados) y fenómenos (experiencias o metáforas)

El poder de estas narraciones se basa en el lenguaje endémico del museo: el lenguaje museográfico. Lenguaje que utiliza dos tipos de activos propios que se caracterizan por su tangibilidad: los objetos y los fenómenos (Wagensberg y Terrades, 2006). A su vez, cada uno de ellos puede emplearse como auténtico o representado, es decir, pueden presentarse a sí mismos o representar a otra cosa o concepto, según se ve en la figura 4. De ahí surgen los cuatro recursos fundamentales del lenguaje museográfico: 


\section{Forma de plasmación}

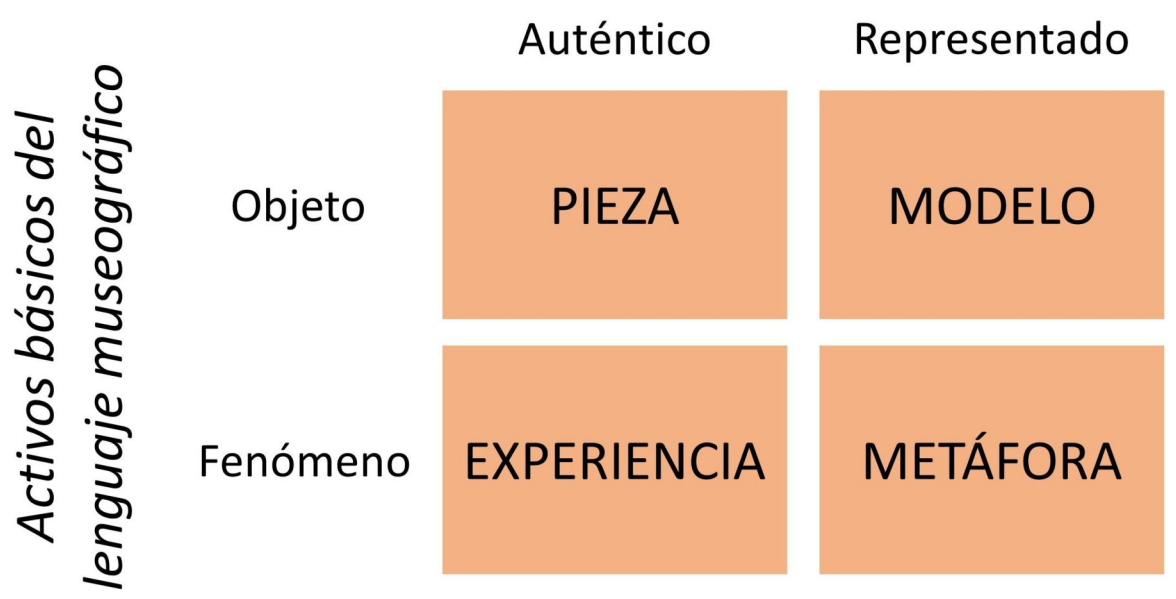

Fig. 4 Recursos básicos del lenguaje museográfico según su forma de plasmación.

Hasta mediado el Siglo XX, se consideraba que los objetos — las piezas de colección—, eran los únicos elementos que podían constituir la base del lenguaje museográfico. El nacimiento de los Science center, rompió esta concepción. En ellos se expusieron toda clase de fenómenos, primero el Palais de la Decouverte en 1932 y el Exploratorium y el Ontario Science Center en 1969 después, supusieron un vuelco de lo hasta entonces establecido.

Los objetos pueden ser auténticos — piezas de colección con significado per se- o representados - maquetas, modelos demostrativos, réplicas, escenografías...-. A su vez los fenómenos también pueden ser auténticos o representados. Los primeros son las experiencias que se presentan a sí mismas y pueden estar relacionadas con la naturaleza - una aurora boreal, una mariposa saliendo de su crisálida..._ o con el ser humano y su actividad —el tañido de una campana, el olor del napalm ardiendo...-. Los segundos representan otros fenómenos como metáforas - sonidos u olores que aportan dimensión fenomenológica a una escenografía, un recipiente lleno de pelotas de pimpón animadas por aire comprimido para ilustrar la composición de un gas...-(Fernández, 2018: p. 64).

Los fenómenos, nacidos en los llamados centros de ciencia, también han sido adoptados museográficamente en museos de arte contemporáneo (Fernández, 2020) aunque, en cualquier caso, este poderoso recurso está en sus inicios de desarrollo, suponiendo una potente línea estratégica de futuro. Rara vez los recursos del lenguaje museográfico aparecen aislados, pues normalmente se emplean en los museos soluciones complejas que implican diversos recursos de modo mixto. 
El poder de estos recursos es su tangibilidad. La sorpresa, la emoción, el vínculo afectivo que producen, las conversaciones que generan son incomparables y esto debe ser tenido muy en cuenta cuando hablamos de iniciativas de digitalización. La pregunta «¿Es real?» nunca se generará delante de una pantalla; por muy bien definida que sea una imagen, tan sólo es una imagen, una representación. No pretendamos trasladar lo tangible a lo representado tal cual. Los proyectos digitales, requieren de contenidos digitales exprofeso, necesitan de una planeación específica. Un objeto digitalizado en 3D nunca podrá suplir emocionalmente al objeto real contemplado in situ. Podrá ofrecer infinitas posibilidades informativas que lo enriquecerán, que lo complementarán, pero no podrá generar el poder experiencial que tiene su contemplación personal. En la sala de exposición el objeto o el fenómeno están presentados, mientras que, en la pantalla, están representados (Fernández, 2018: p. 63). Así pues, en la iniciativa digital que se quiera emprender, la historia que se quiera explicar adquiere todavía más valor si cabe, para que tenga éxito.

Sin historia, sin una buena historia no hay exposición ni recurso digital. Historia que debe estar vinculada a las historias personales de los visitantes presenciales o virtuales, que deben establecer este vínculo emocional. Seamos claros. No existe la exposición virtual, no existe la museografía virtual al igual como no existe la gastronomía virtual. Llamar así a lo que tan sólo son recursos — ¡muy potentes! — del sitio web del museo, no favorece el papel social del museo, sino que, a nuestro parecer, lo limita.

Los recursos digitales son infinitos y en desarrollo constante. Pretender estar al día de todas las novedades que salen es imposible y más con la escasez crónica de recursos de la que adolecen los museos españoles y de muchos más países. Asimismo, debemos tener en cuenta que muchas de las novedades tecnológicas que se anunciaron como revolucionarias con la digitalización han resultado en fiascos —el láser disc, el cine 3D, las Google glass...- o no han experimentado el desarrollo tan rápido o hegemónico como se preveía - el reconocimiento de texto, el DNI electrónico, la realidad virtual, el libro electrónico...-.

Resumiendo, las narrativas específicas que los recursos del lenguaje museográfico nos ofrecen, son las que nos deben ayudar a construir los proyectos de digitalización que queramos emprender, como bien dice el director del Museo Nacional de Arte de Catalunya, Pepe Serra (Tramullas, 2020): «Algunos museos estábamos intentando cambiar de paradigma - explica-, dejar de describir las cosas que tenemos y pasar a narrarlas, explicar historias individualizadas, que vinculen a las personas con el museo. Del mismo modo como también deberemos tener claras las narrativas que queremos ofrecer para que el nuestro sea un museo social, participativo y comunitario». Es decir, las narrativas deben ser transversales para que lo que hagamos a nivel de digitalización, sirva para la transformación del museo hacia una institución mucho más social, mucho más participativa, mucho más en contacto con su comunidad y no sólo para llegar a números más altos de visitantes, aunque este sea un objetivo lícito. 


\subsection{Una guía para la evaluación previa de iniciativas de digitalización.}

Una de nuestras líneas de actuación es la de proveer un repositorio de recursos para los museos. Hasta el momento, este repositorio contenía artículos, libros, vídeo o audios. Con la guía que presentamos iniciamos una nueva vía de desarrollo con materiales elaborados exprofeso con voluntad de servir de ayuda en la gestión estratégica de los museos.

El proceso de elaboración de la guía ha contado con la colaboración desinteresada de diversas personas con las que nos pusimos en contacto. Cuatro de ellas, adheridas a nuestro manifiesto ${ }^{1}$ —Eloisa Pérez, Albert Díaz, Óscar Menéndez y Artur Moreno-y otras dos que por su experiencia creímos necesario consultar — Susana Funes (NETSU) y Nacho Granero (La Cultura Social) - . A todas ellas queremos agradecer la riqueza de sus aportaciones que no sólo han mejorado mucho la versión inicial, sino que además nos han obligado a reflexionar como colectivo.

Empezamos con la identificación de los criterios que deberían ser tenidos en cuenta en la valoración. Decidimos agruparlos en dos grandes apartados: vínculo museístico y gestión ejecutiva.

Según el primero, un proyecto debería estar directamente relacionado con los contenidos del museo conforme a los criterios siguientes:

- El vínculo con la función educativa o comunicativa del museo

- El vínculo con el contenido propio.

- El fomento de la experiencia vivencial del participante.

- El fomento de la implicación activa del participante.

- La socialización.

- La identificación del público diana al que se dirige.

En cuanto a la gestión ejecutiva, los criterios a seguir son:

- Si dispone de los recursos financieros, humanos y materiales adecuados.

- Si la tecnología empleada tiene posibilidades de desarrollo y no corre el riesgo de quedar obsoleta con facilidad.

- Si su desarrollo ha contemplado la participación interna.

- Si prevé una evaluación sistemática y formativa que garantice la adecuación a lo largo del tiempo.

Estos criterios fueron convertidos en indicadores con tres niveles de desempeño: inapropiado, correcto o idóneo. Para cada indicador, los niveles de desempeño se describieron con un redactado preciso intentando huir de toda ambigüedad.

\footnotetext{
${ }^{1}$ https://www.elmuseotransformador.org/manifiesto/
} 
Una vez realizada una primera versión de la rúbrica, la pasamos a consulta de las personas mencionadas anteriormente y a partir de sus comentarios y observaciones, hicimos la versión final que presentamos en el congreso.

\section{Resultados}

Después de todo el proceso de consulta, la guía, (descargable de forma gratuita en https://www.elmuseotransformador.org/recursos-evaluacion/), se ha diseñado en tres partes:

- Introducción. La estrategia

- Rúbrica de evaluación.

- Mapa de ubicación.

Por sus características, la guía puede ser aplicada tanto a proyectos más globales como a actividades muy concretas. No se pretende que sea un estándar igual para todos ni devenir un recetario, sino lo que su nombre indica: una guía para ayudar en la toma de decisiones.

El segundo de los objetivos que nos hemos propuesto es el de «garantizar un proceso de reflexión transversal que implique a todos los departamentos». Si queremos un museo transformador de nuestro entorno, debemos incorporar la participación de las personas que configuran la plantilla del museo. No podremos ser nunca un museo con impacto social si no somos un museo que tenga entre sus líneas básicas el de la participación interna. Es necesario evidenciar si lo que quiere desarrollar la organización está en consonancia con lo que internamente se considera necesario y útil. Así pues, en la reflexión sobre la rúbrica deberían tomar parte el máximo de personas posible y siempre, aquellas que directa o indirectamente se vean concernidas.

Igualmente, la guía no es el único instrumento que debe ser tenido en cuenta, tan sólo es uno más. Conversaciones, encuestas, talleres, entrevistas, etc. pueden y deben formar parte de un proceso más o menos complejo en función de la dimensión de la propuesta.

\subsection{Introducción. La estrategia}

La digitalización del museo debe ser un proceso estratégico, no coyuntural. Así pues, la guía propone un proceso de trabajo que empieza por unas preguntas clave que se deberían responder en cualquier innovación que el museo quiera implantar, sea digital o no.

A veces se arguye que muchas propuestas innovadoras surgen de ideas que no estaban contempladas en los planes específicos pero que deben ser llevadas a cabo, aunque sea en plan de experimentación ya que la investigación también debe ser tenida en cuenta en este campo. De acuerdo, pero creemos que este planteamiento puede confundir investigación con intentonas sin fundamento $\mathrm{u}$ ocurrencias varias y no es el caso. Cuando se definen los objetivos estratégicos que deben desarrollar la misión del museo, no se explicitan acciones concretas sino propósitos de llegada. Es posteriormente que deben definirse las acciones para que estos objetivos puedan llegar a ser alcanzados. Y si se detectan déficits en los planes 
estratégicos, se supone que estos deberían estar en constante evaluación para adecuarse a la realidad cambiante.

Siguiendo este razonamiento, cualquier iniciativa de digitalización, debería empezar con la respuesta a tres preguntas estratégicas según puede verse en el esquema de la figura 5:

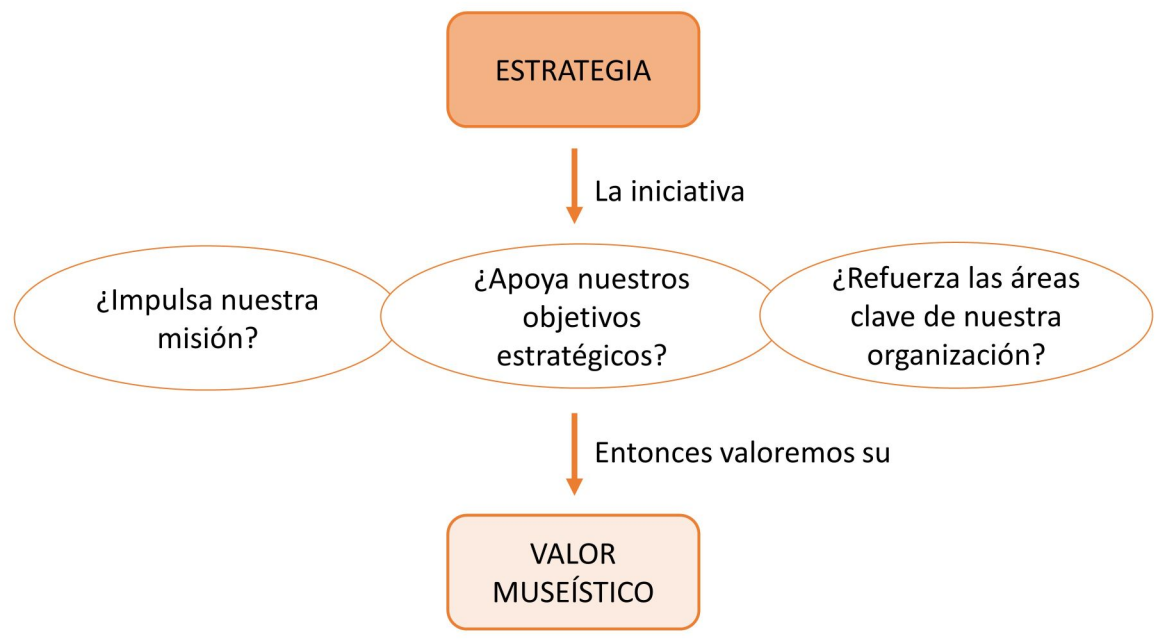

Fig. 5 Proceso de toma de decisiones. De la estrategia al valor museístico

Es en función de la misión del museo y de sus objetivos estratégicos que debe valorarse la toma en consideración o no de una nueva estrategia digital. Podríamos decir que esta primera parte tendría carácter eliminatorio.

En nuestra guía, estas preguntas no están formuladas en forma de indicadores con sus niveles de desempeño redactados ya que, a nuestro parecer, son tres preguntas excluyentes. Si la respuesta a estas tres preguntas es negativa, la iniciativa no debería ser tenida en cuenta. La respuesta positiva a una de las preguntas, si la planificación estratégica es coherente, debe conllevar el mismo signo de respuesta a las otras dos. No tendría ningún sentido que consideráramos que la iniciativa impulsa nuestra misión, y en cambio no apoyara los objetivos estratégicos o reforzara áreas clave.

Pero para poder valorar con más detenimiento este apartado, puede ser también útil utilizar otras herramientas que no descartamos desarrollar en un futuro. Será el museo el que decida si las quiere usar y cómo. Grupos de discusión o comunidades de práctica en el campo de la cooperación o, en un plano más concreto, utilizar un modelo canvas adaptado al entorno museístico, pueden ser estrategias que aseguren un desarrollo correcto de cualquier iniciativa. 


\subsection{Rúbrica de evaluación}

Una vez superada la primera fase, entramos propiamente en la rúbrica que nos permitirá bajar a la concreción. La primera parte, analiza el valor museístico de la propuesta como concreción táctica de los objetivos estratégicos como se ve en la figura 6 .

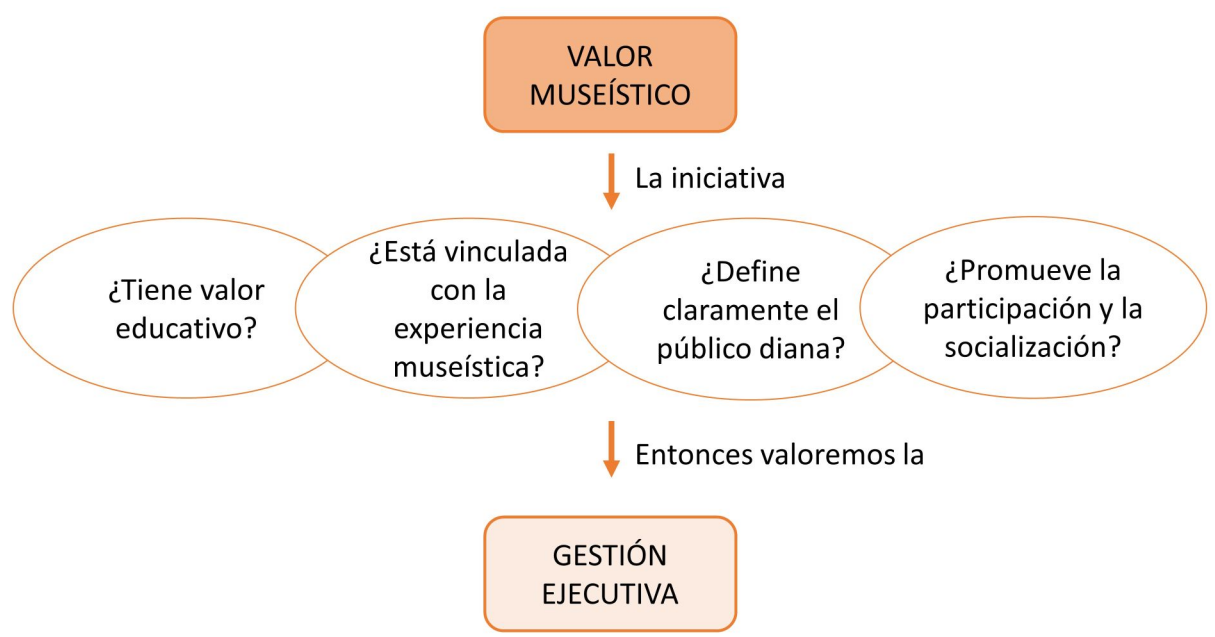

Fig. 6 Proceso de toma de decisiones. Del valor museístico a la gestión ejecutiva

Entendemos por valores museísticos todos aquellos que se refieren a su valor educativo o divulgativo, al vínculo directo con los contenidos propios del museo, sean las exposiciones, las colecciones, la investigación, etc., a la definición precisa de un público diana y a la promoción de la socialización y la implicación activa del participante.

Es obvio que intentar recoger todas las variables posibles en una única rúbrica es imposible. Viene aquí pues, el papel de un museo determinado en adaptar esta rúbrica a sus necesidades concretas. Un proyecto para digitalizar piezas de la colección en 3D, no tiene una repercusión directa en valores educativos o de investigación, pero sí debería tenerla a partir de su uso por otras plataformas en las que se vuelquen. Es decir, la mera digitalización no tiene sentido si el museo no se plantea está dentro de un proyecto más global.

Una vez valorado el valor museístico de la propuesta, es el momento de ver si su gestión puede ser llevada a cabo con los recursos disponibles o asignados, para lo que hemos planteado la segunda parte que responde a las preguntas del esquema de la figura 7 : 


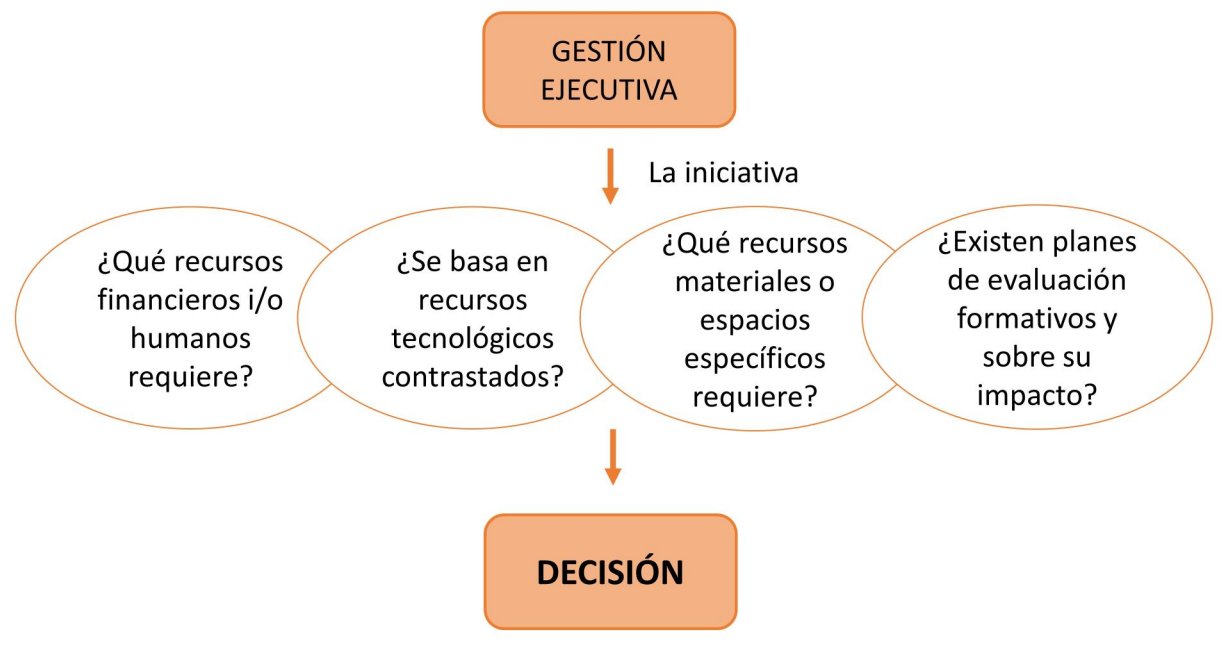

Fig. 7 Proceso de toma de decisiones. De la gestión ejecutiva a la decisión final

Hemos partido del valor estratégico de la propuesta para concretar su valor museístico. Ha llegado el momento ahora de valorar la gestión ejecutiva necesaria. En este apartado de la rúbrica tendremos que valorar si los recursos financieros y humanos necesarios son adecuados, si los recursos tecnológicos que requerirá están contrastados, los materiales y espacios físicos específicos que requerirá y los mecanismos de evaluación formativa con que nos dotaremos para ir adecuando su desarrollo.

El formato final de la rúbrica es el de la figura 8: un cuadro de doble entrada en el que en las filas se desarrollan los indicadores y en las columnas los niveles de desempeño.

\begin{tabular}{|c|c|c|c|c|}
\hline Indicador & Inapropiada & Correcta & Idónea & Puntos \\
\hline Valor & -1 & 0 & +1 & \\
\hline \multicolumn{5}{|c|}{ Valor museístico } \\
\hline $\begin{array}{l}\text { Vínculo con la } \\
\text { función } \\
\text { educativa del } \\
\text { museo } \\
\end{array}$ & $\begin{array}{l}\text { La actividad no ejerce ningún papel educativo ya } \\
\text { que no genera ninguna transformación intelectual } \\
\text { en el usuario bien sea de tipo experiencial, } \\
\text { estético o de aprendizaje. }\end{array}$ & $\begin{array}{l}\text { La actividad tiene algún valor educativo ya que } \\
\text { promueve la acción del participante que genera } \\
\text { cambios intelectuales experienciales, estéticos o } \\
\text { de aprendizaje. }\end{array}$ & $\begin{array}{l}\text { La actividad es totalmente educativa ya que la } \\
\text { acción del participante pretende una } \\
\text { transformación intelectual de la persona que aúna } \\
\text { experiencia, estética y aprendizaje }\end{array}$ & \\
\hline $\begin{array}{l}\text { Vínculo con el } \\
\text { contenido del } \\
\text { museo }\end{array}$ & $\begin{array}{l}\text { La actividad podría ser impulsada por una } \\
\text { institución ajena al museo ya que no tiene ningún } \\
\text { vínculo con los contenidos propios del mismo. }\end{array}$ & $\begin{array}{l}\text { La actividad sólo puede ser desarrollada por el } \\
\text { museo ya que se vincula directamente con sus } \\
\text { contenidos, aunque esto no queda bien explicitado } \\
\text { en su desarrollo. }\end{array}$ & $\begin{array}{l}\text { La actividad no la podría llevar a cabo ninguna otra } \\
\text { institución ya que se vincula manifiestamente con } \\
\text { los contenidos del museo y su desarrollo lo } \\
\text { muestra de forma explicita. }\end{array}$ & \\
\hline $\begin{array}{l}\text { Vínculo con la } \\
\text { experiencia } \\
\text { museistica }\end{array}$ & $\begin{array}{l}\text { La actividad no remite en modo alguno a la visita } \\
\text { presencial, ni apela a sus activos. No existe ningún } \\
\text { vinculo entre lo digital y lo presencial. }\end{array}$ & $\begin{array}{l}\text { La actividad tiene su desarrollo principal online, } \\
\text { aunque estimula la visita presencial al museo con } \\
\text { los activos sociales e intelectuales del contacto } \\
\text { tangible con los elementos museísticos: objetos y } \\
\text { fenómenos. }\end{array}$ & $\begin{array}{l}\text { La actividad apela sistemáticamente a los activos } \\
\text { singulares de la visita presencial al museo, y a la } \\
\text { particular repercusión intelectual de la experiencia } \\
\text { museística en las salas (objetos y fenómenos } \\
\text { tangibles), con el fin de priorizar y promocionar la } \\
\text { visita presencial. }\end{array}$ & \\
\hline $\begin{array}{l}\text { Participación } \\
\text { de las } \\
\text { personas }\end{array}$ & $\begin{array}{l}\text { La actividad es totalmente pasiva, no requiere } \\
\text { ningún ejercicio activo de las personas que asisten } \\
\text { a ella, limitándose al manejo del hardware } y \\
\text { software requeridos. }\end{array}$ & $\begin{array}{l}\text { La actividad requiere de cierta participación de las } \\
\text { personas que asisten a ella aunque se limita al } \\
\text { planteamiento de preguntas o cuestiones que no } \\
\text { implican una intervención esencial en ella. }\end{array}$ & $\begin{array}{l}\text { La actividad está planteada para favorecer la } \\
\text { participación productiva de las personas que } \\
\text { colaboran en ella que aportan elementos que la } \\
\text { enriquecen en un proceso de co-creación activa. }\end{array}$ & \\
\hline $\begin{array}{l}\text { Promoción de } \\
\text { la socialización }\end{array}$ & $\begin{array}{l}\text { La actividad está planteada para el disfrute de un } \\
\text { individuo aislado sin necesidad de compartirla con } \\
\text { otras personas de su entorno familiar o } \\
\text { convivencial. }\end{array}$ & $\begin{array}{l}\text { La actividad se puede desarrollar de forma } \\
\text { individual o colectiva por parte de los participantes } \\
\text { aunque su desarrollo no promueve el encuentro } \\
\text { de las personas del entorno familiar o convivencial. }\end{array}$ & $\begin{array}{l}\text { La actividad ha sido diseñada con la intención de } \\
\text { promover y facilitar su realización de forma } \\
\text { compartida por un grupo de personas del entorno } \\
\text { familiar o convivencial. }\end{array}$ & \\
\hline $\begin{array}{l}\text { Público al que } \\
\text { se dirige la } \\
\text { actividad }\end{array}$ & $\begin{array}{l}\text { La actividad se dirige a un público minoritario y } \\
\text { con alto nivel cultural ya que requiere de } \\
\text { conocimientos especificos sobre el tema. }\end{array}$ & $\begin{array}{l}\text { La actividad se dirige a un público general en el } \\
\text { que no es necesario tener conocimientos } \\
\text { especificos sobre el tema más que una cultura } \\
\text { general aunque se dirige a una edad determinada. } \\
\text { Tiene en cuenta algunos aspectos de accesibilidad. }\end{array}$ & $\begin{array}{l}\text { La actividad está diseñada para que cualquier } \\
\text { persona de cualquier edad pueda participar en ella } \\
\text { y tiene en cuenta los aspectos de accesibilidad. }\end{array}$ & \\
\hline & & & Total & \\
\hline
\end{tabular}

Fig. 8 Formato de la rúbrica 
En la primera columna de cada fila se define el indicador a valorar. Las tres siguientes explicitan las descripciones de desempeño: inapropiada, correcta e idónea. Una quinta columna sirve para anotar la puntuación obtenida para cada indicador: -1 si es inapropiada, 0 si es correcta $\mathrm{o}+1$ si es idónea. Al final de los dos apartados de la rúbrica se anota la suma de valores que nos dará la puntuación final de cada uno de ellos.

\subsection{Mapa de ubicación}

Pero para facilitar un poco más la toma de la decisión, hemos añadido un mapa de coordenadas donde se puede ubicar la propuesta según la puntuación obtenida (ver figura 9):

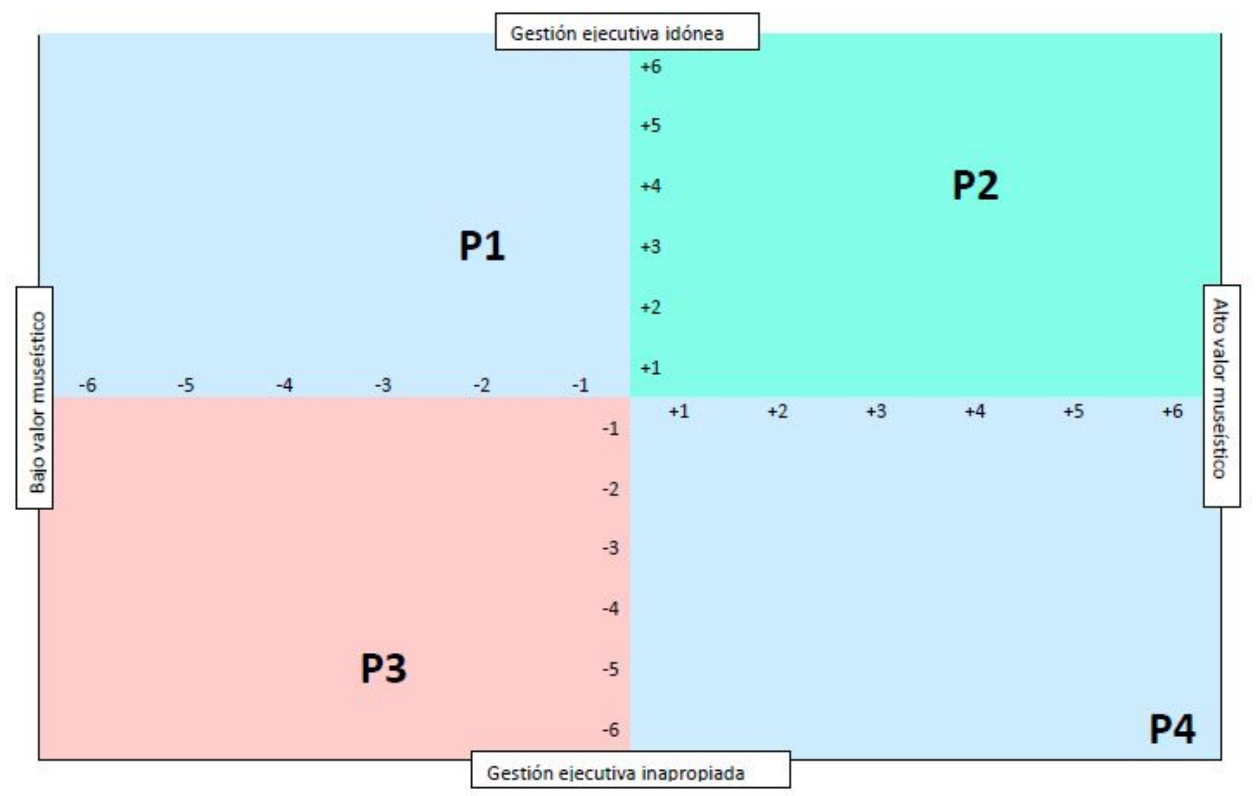

Fig. 9 Mapa de ubicación de las propuestas

El eje de abscisas representa la puntuación obtenida en el apartado de valor museístico mientras que el de ordenadas el de gestión ejecutiva. Después de aplicar la rúbrica, una propuesta que se ubicara en el cuadrante superior derecha sería apropiada y cuanto más alejada del 0, mejor. En cambio, una propuesta que se ubicara en el cuadrante inferior izquierda debería hacer reflexionar mucho sobre su toma en consideración. Los otros dos cuadrantes contendrían aquellas propuestas factibles pero que requerirían o bien un replanteamiento de la propia iniciativa o bien una revisión de los valores museísticos que conlleva o de su ejecución ejecutiva.

La rúbrica y este mapa de ubicación, como ya se ha especificado, son tan sólo una herramienta de ayuda para la toma de decisiones, sin que representen una receta que deba ser tomada en cuenta al pie de la letra. Es una herramienta dinámica de manera que cada museo debe hacérsela suya y adaptarla a sus características específicas. Dado que todavía no ha 
podido ser probada en la práctica, cualquier feedback que se reciba, servirá para mejorarla y así, incrementar las posibilidades de ayuda a otros museos.

\section{Conclusiones}

La gestión de los museos adolece en la mayor parte de los casos, de un déficit grande de proyectos de evaluación, lo cual en última instancia procede de los déficits de planificación estratégica. Un síntoma claro de que no existe una gestión estratégica eficaz es que las labores de evaluación se juzgan algo innecesario, superfluo o incluso ostentoso. Se suele actuar más por intuición, opinática o incluso por capricho de directivos, de políticos o de presidentes de patronato, que por un análisis estratégico de las necesidades reales del museo. Necesidades que deben tener en cuenta que un museo debe ser una institución socialmente comprometida en la conservación y educación que comunica su patrimonio a través del lenguaje museográfico.

El auge de las propuestas digitales de los museos a lo largo de la pandemia puede llevar a la falsa creencia de que este es el camino a seguir. Compartimos los miedos que expresa Brenda J. Caro (2020): «Me gustaría pensar que esta exigencia viene urgida por una necesidad de repensar el carácter social del museo y sus propias inercias institucionales respecto al tipo de espacio público y de esfera pública que construye. Sin embargo, me temo que el viraje y la "demanda" por trasladarse a lo digital deviene de otro tipo de lógicas y políticas económicas que se han vuelto parte de la política cultural gubernamental, universitaria y privada, y que se han presentado como paradigma y deber ser inevitable de toda institución que se respete».

Creemos que, en muchos casos, este hecho se da por la inexistencia de un corpus de recursos de gestión adecuados a las labores propias del museo. Muy a menudo se han adaptado recursos de otros ámbitos sin que haya habido una verdadera reflexión sobre su adecuación a las funciones propias del museo. Especialmente en el campo del márquetin donde se han adoptado sin adaptarlos de forma conveniente. Así, en lugar de adaptar el márquetin al museo, en ocasiones se ha acabado adaptando el museo al márquetin.

Con esta propuesta que no queremos presentar cerrada sino abierta a los cambios necesarios, pretendemos aportar un pequeño grano de arena para paliar este déficit. Puede ser tomada como herramienta que se utilice tal cual, pero también como elemento de reflexión sobre el proceso que debería seguir la toma de decisiones en cualquier iniciativa de digitalización que se pretenda llevar a cabo en un museo. Y por extensión, valoramos que puede ser adaptada a cualquier otro ámbito de desarrollo en la gestión estratégica del museo ante iniciativas nuevas, fomentando la conversación, la transversalidad y el trabajo cooperativo en el seno del personal del museo.

Esperamos haber podido contribuir, cuando menos, a generar el debate sobre la necesidad de pensar estratégicamente antes de actuar ejecutivamente. 


\section{Referencias}

BROCKËR B. (2021). "A Race to the Bottom: Why Museums Need a Digital Strategy» en Rolling Stone. $\quad<$ https://www.rollingstone.com/culture-council/articles/race-to-the-bottom-museumsdigital-strategy-1111400/> [Consulta: 15 de enero de 2021].

CARO, B.J. (2020). «Conectarse, desconectarse, volver a conectar: el museo lanzado hacia lo digital» en Terremoto. Contemporary Art in The Americas. <https://terremoto.mx/online/conectarsedesconectarse-volver-a-conectar-el-museo-lanzado-hacia-lo-digital/> [Consulta: 25 de julio de 2020].

FERNÁNDEZ, G. (2018). El museo de ciencia transformador. Un ensayo a favor de la relevancia social del museo de ciencia contemporáneo. Tarragona: nuestrasnubes.com $<$ https://www.elmuseodecienciatransformador.org > [Consulta: 24 de enero de 2021].

FERNÁNDEZ, G. (2020). «Colecciones de fenómenos (y no sólo de objetos) en los museos» en El Museo Transformador. $<$ https://www.elmuseotransformador.org/colecciones-de-fenomenos-y-nosolo-de-objetos-en-los-museos/> [Consulta: 2 de febrero de 2021].

LÓPEZ, F. (2021). «Esperando unos gloriosos años 20... la magnitud de la tragedia III» en Teknecultura <https:/teknecultura.com/bloc/esperando-unos-gloriosos-anos-20-la-magnitud-dela-tragedia-iii/> [Consulta: 25 de enero de 2021].

SIMON, N. (2020). «How Can I Contribute? Four Steps I'm Taking to Figure it Out» en Medium $<$ https://medium.com/@ninaksimon/how-can-i-contribute-four-steps-im-taking-to-figure-it-outd7a35b5149d5> [Consulta: 16 de junio de 2020].

STENGLER, E.; et al. (2020). «Después de la crisis del coronavirus... ¿los museos volverán a abrir?» en Nodocultura <https://nodocultura.com/2020/04/03/los-museos-volveran-a-abrir/> [Consulta: 20 de diciembre de 2020].

TRAMULLAS, G. (2020). «Los museos, hacia una mayor convivencia física y digital» en Cuaderno $<$ https:/www.elperiodico.com/es/cuaderno/20200328/futuro-museos-tras-crisis-coronavirus7906604> [Consulta: 24 de noviembre de 2020].

WAGENSBERG, J. y TERRADES ARCHITECTS (2006). El museo total. Barcelona: Sacyr. 